\title{
Induced Unbalanced Linguistic Ordered Weighted Average
}

\author{
Lucas Marin $^{1}$ José M. Merigó $^{2}$ Aida Valls ${ }^{1}$ Antonio Moreno ${ }^{1}$ David Isern ${ }^{1}$ \\ ${ }^{1}$ Universitat Rovira i Virgili, Departament d'Enginyeria Informàtica i Matemàtiques, ITAKA Research Group. \\ Av. Països Catalans, 26, 43007 Tarragona, Catalonia (Spain) \\ ${ }^{2}$ University of Barcelona, Department of Business Administration, Av. Diagonal 690, 08034 Barcelona, Catalonia
} (Spain)

\begin{abstract}
Aggregation operators for linguistic variables usually assume a uniform and symmetrical distribution of the linguistic terms that define the variable. This paper defines the Induced Unbalanced Linguistic Ordered Weighted Average (IULOWA). This aggregator takes into account the fuzzy membership functions of the terms during the aggregation operations of the pairs of terms. There is no restriction on the form of the membership functions of the terms, which can be triangular or trapezoidal, non-symmetrical and non-equally distributed. Moreover, the paper proposes to use the specificity and fuzziness measures of the terms to induce the order of the arguments, providing some examples of this criterion in decision making.
\end{abstract}

Keywords: Aggregation operator; Unbalanced fuzzy sets; Order inducing variables; Linguistic variables.

\section{Introduction}

Aggregation operators are very common in the literature [1-3]. They are mathematical functions that reduce a set of values into a unique representative one that summarizes the inputs, in some way. They are useful in a wide range of applications including statistics, economics and engineering. An interesting aggregation operator is the ordered weighted averaging (OWA) operator [4]. It introduces an ordering of the values of the arguments before being aggregated according to some combination policy. In fact, it provides a parameterized family of aggregation operators between the minimum and the maximum.

In decision making, an interesting extension of the OWA operator is the induced OWA (IOWA) operator [5]. Its main difference is that it uses a reordering process in the aggregation based on order-inducing variables. Thus, it is able to consider an additional reordering criterion that does not depend on the values of the arguments. Since its appearance, it has been studied by many authors (e.g. [6-8] developed several extensions for group decision making problems, and [9] makes a generalisation based on quasi arithmetic means).

Although it is commonly assumed that we operate with numbers, we may find many applications where the available information cannot be assessed with numerical values. In this case, it is more appropriate to make use of linguistic variables. The use of linguistic assessments permits to deal with the information in a qualitative way. Most of the studies in this direction have assumed a uniform and symmetrical distribution of the linguistic terms that define the linguistic variable [10]. However, there are some situations that cannot be modelled with symmetric linguistic variables. Therefore, some authors $[11,12]$ have recently suggested the use of unbalanced linguistic variables because they give more flexibility in the definition of the linguistic terms (see Fig 1). Recently, some unbalanced aggregation operators have appeared $[13,14]$.

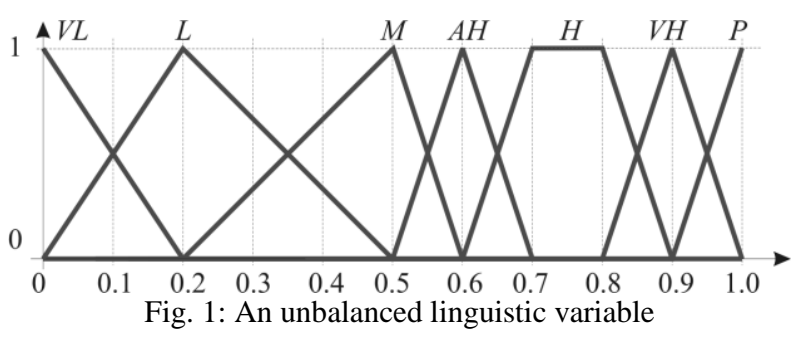

This paper is a step forward to suggest new unbalanced linguistic aggregation operators such as the induced unbalanced linguistic ordered weighted averaging (IULOWA) operator. It is an aggregation operator that provides a parameterized family of aggregation operators between the linguistic minimum and maximum. It is based on the definition of the ULOWA made by the authors in [15], but including order-inducing variables in the aggregation. The paper shows that IULOWA fulfils the monotonicity, identity, symmetry and boundary conditions, usually required to aggregation operators.

The second important contribution of this paper is the proposal of using some of the information related to the definition of the labels as order-inducing criterion in IULOWA. In this sense, the arguments are decreasingly ordered by the degree of uncertainty of the labels, giving priority to more specific values as they represent more precise information given by the decision-maker.

The rest of the paper is organised as follows. Section 2 provides some basic preliminaries. Section 3 defines the IULOWA operator and studies its properties. Section 4 proposes how to make use of the different degrees of uncertainty in the unbalanced terms as orderinduction criterion. Section 5 develops several illustrative examples of the IULOWA operator with the proposed order induction. Finally, section 6 gives the main conclusions of the paper and suggests some lines of future work. 


\section{Preliminaries}

\subsection{Linguistic variables}

Let us consider a set of linguistic labels $S=\left\{s_{i}\right\}, i \in\{0, \ldots, T\}$. This set $S$ is defined as a finite and totally ordered term set on a reference domain $X=[0,1]$, with an odd cardinal, where one of the labels corresponds to the neutral value and the remaining terms are placed around it $[12,16,17]$. The cardinality of the set must be small enough so as not to impose useless precision and rich enough in order to allow an appropriate discrimination level. The usual cardinality values are 7 or 9.

The semantics of each linguistic label is given by a trapezoidal or triangular membership function $\mu: X \rightarrow[0,1]$, that is represented with a tuple $P=\left(p_{1}, p_{2}, p_{3}, p_{4}\right)$, where $p_{1}<p_{2}<p_{3}<p_{4}$ are the points in the reference domain $X$ which define the trapezoid. Some special cases can be defined. If $p_{1}=p_{2}$ and $p_{3}=p_{4}$ then $P$ corresponds to a crisp interval. If $p_{2}=p_{3}$ the fuzzy set $P$ is triangular. If $p_{1}=p_{2}=p_{3}=p_{4}$, then $P$ is called a crisp real number.

\subsection{ULOWA: Unbalanced Linguistic Ordered Weighted Average}

The ULOWA operator was defined in [15] as an extension of the LOWA operator [17], in order to deal with unbalanced linguistic terms.

Let $A=\left\{a_{1}, \ldots, a_{m}\right\}$ be a set of labels, where $a_{j} \in S$, that have to be aggregated with respect to a set of weights $W=\left(w_{1}, \ldots, w_{m}\right)$ such that $w_{i} \in[0,1]$ and

$\sum w_{i}=1$. Those weights determine the decision-maker policy, so that we can emphasize different arguments based upon their ordered position.

The ULOWA operator is defined as:

$$
\begin{aligned}
& U L O W A_{W}\left(a_{1}, \ldots, a_{m}\right)=W \cdot B^{T}= \\
& =C^{m}\left\{w_{k}, b_{k}, k=1, . ., m\right\}= \\
& =w_{1} \otimes b_{1} \oplus\left(1-w_{1}\right) \otimes C^{m-1}\left\{\beta_{h}, b_{h}, h=2, . ., m\right\}
\end{aligned}
$$

In this definition $\beta_{h}=w_{h} / \sum_{2}^{m} w_{h}, h=\{2, \ldots, m\}$ and $B=\left\{b_{1}, \ldots, b_{m}\right\}$ is a permutation of the elements of $A$, such that $B=\sigma(A)=\left\{a_{\sigma(1)}, \ldots, a_{\sigma(m)}\right\}, \quad$ where $a_{\sigma(j)} \leq a_{\sigma(i)} \forall i \leq j$.

$C^{m}$ is the convex combination operator of $m$ labels. If $\exists j \in 1 . . m w_{j}=1$ and $\forall k \in 1 . . m, k \neq j w_{k}=0$, then $C^{m}\left\{w_{i}, b_{i}, i=1, . ., m\right\}=b_{j}$.

When $m=2$, the convex combination of the two terms $b_{1}=s_{j}$ and $b_{2}=s_{i}$, with $s_{j}, s_{i} \in S(j \geq i)$ is calculated taking into account the membership functions of labels $s_{j}$ and $s_{i}$.

$$
C^{2}\left\{w_{i}, b_{i}, i=1,2\right\}=w_{1} \otimes s_{j} \oplus\left(1-w_{1}\right) \otimes s_{i}=s_{k}
$$

such that,

$$
k=\underset{i \leq p \leq j}{\arg \max }\left\{\operatorname{Sim}\left(s_{p}, \delta\right)\right\}
$$

Being $x_{s_{i}}^{*}$ the center of gravity of the fuzzy set associated to the label $s_{i}$, then, $\delta$ is an intermediate crisp number defined as $\delta=\left(x_{k}, x_{k}, x_{k}, x_{k}\right) \quad$ with $x_{k}=x_{s_{i}}^{*}+w_{1}\left(x_{s_{j}}^{*}-x_{s_{i}}^{*}\right)$.

The similarity function of two fuzzy sets proposed after a study of the literature [15] is the following one:

$$
\operatorname{Sim}(P, Q)=\left[\sqrt[4]{\prod_{i=1}^{4}\left(2-\left|p_{i}-q_{i}\right|\right)}-1\right]
$$

\subsection{IOWA: Induced OWA}

In [5] the concept of induced ordering was presented. This is a more general type of aggregation that takes into account the ordering that an additional variable is inducing on the set of values to be aggregated. The IOWA operator is defined by as follows:

$$
\operatorname{IOWA} A_{W}\left(\left\langle u_{1}, a_{1}\right\rangle,\left\langle u_{2}, a_{2}\right\rangle, \ldots,\left\langle u_{m}, a_{m}\right\rangle\right)=\sum_{j=1}^{m} w_{j} b_{j}
$$

where $W=\left(w_{1}, \ldots, w_{m}\right)$ is the usual weighting vector that defines the aggregation policy of the OWA operator, with $w_{i} \in[0,1], \sum w_{i}=1$. Then, $b_{j}$ is the $a_{i}$ value of the pair $\left\langle u_{i}, a_{i}\right\rangle$ having the $j$-th largest $u_{i}$.

An important aspect of the IOWA operator is the fact that the ordering induced by the variable $u$ can produce ties in some arguments. In this case, the relative order of two arguments $a_{i}$ and $a_{j}$ with $u_{i}=u_{j}$ is relevant because they may correspond to different values, that is $a_{i} \neq a_{j}$. In many papers the solution taken is substituting $a_{i}$ and $a_{j}$ by their arithmetic average $\left(a_{i}+a_{j}\right) / 2$. Another mechanism to solve the ties consists in including a secondary ordering criterion [7].

\section{The induced ULOWA operator}

\subsection{Introduction}

The induced unbalanced LOWA (IULOWA) is an aggregation operator for linguistic values that are defined on an unbalanced vocabulary $S$. As IOWA, the operator is able to assess complex decision problems based on the use of order inducing variables.

Definition 1: The Induced Unbalanced Linguistic Ordered Weighted Average, based on the ordering criterion $u$, is calculated as:

$$
\begin{aligned}
& I U L O W A_{w}\left(\left\langle u_{1}, a_{1}\right\rangle,\left\langle u_{2}, a_{2}\right\rangle, \ldots,\left\langle u_{m}, a_{m}\right\rangle\right)= \\
& W \cdot B^{T}=C^{m}\left\{w_{k}, b_{k}, k=1, \ldots, m\right\}= \\
& w_{1} \otimes b_{1} \oplus\left(1-w_{1}\right) \otimes C^{m-1}\left\{\beta_{h}, b_{h}, h=2, \ldots, m\right\},
\end{aligned}
$$

where $B$ is the induced ordered vector, i.e., $B=\left(a_{\sigma(1)}^{\prime}, a_{\sigma(2)}^{\prime}, \ldots, a_{\sigma(m)}^{\prime}\right)$ where $a_{\sigma(j)}^{\prime}$ corresponds to 
the value $a_{j}$ having the $j$-th largest $u_{i}$. $W=\left(w_{1}, \ldots, w_{m}\right)$ is the usual weighting vector that defines the aggregation policy of the OWA operator, with $w_{i} \in[0,1], \sum w_{i}=1$. The final convex combination of two linguistic terms is the same as in the ULOWA operator defined before in Eq. 2.

This operation consists on ordering the values of the arguments according to the inducing variable $u$, and then applying the ULOWA operator defined before in Eq. 1.

Note that following the OWA literature $[3,6,18,19]$ we could study many measures for characterizing the IULOWA operator such as the distinction between descending and ascending orders, the use of mixture aggregations and the use of infinitary aggregations.

\subsection{Properties}

As stated in [4], an OWA operator should satisfy the following properties: monotonicity, commutativity, idempotency, and to be a bounded operator (i.e. "orand").

Property 1: The IULOWA operator is increasingly monotonous with respect to the argument values if the associated order inducing values remain unchanged:

Let us consider two order-induced vectors $A=\left\{\left\langle u_{1}, a_{1}\right\rangle, \ldots .,\left\langle u_{m}, a_{m}\right\rangle\right\}$ and $B=\left\{\left\langle u_{1}^{\prime}, b_{1}\right\rangle, \ldots .,\left\langle u_{m}^{\prime}, b_{m}\right\rangle\right\}$, such that $\forall j, u_{j}=u_{j}^{\prime} \quad$ and $\forall j, a_{j} \geq b_{j}$, then $\operatorname{IULOWA}(A) \geq I U L O W A_{w}(B)$.

That means, as will be detailed in section 4 , that if we substitute each term by another that it has the same specificity and fuzziness but has a greater preference in the scale $S$, the result will also be a term equal or better in the preference scale. In fact, this case reduces the proof to the ULOWA operator [15] because the inducing variable does not change the order.

Proof: Let $\operatorname{IULOWA}_{w}(A)=I U L O W A_{w}\left(\left\langle u_{1}, a_{1}\right\rangle, \ldots,\left\langle u_{m}, a_{m}\right\rangle\right)$, and $\operatorname{IULOWA}_{w}(B)=I U L O W A_{w}\left(\left\langle u_{1}^{\prime}, b_{1}\right\rangle, \ldots .,\left\langle u_{m}^{\prime}, b_{m}\right\rangle\right)$. If $\forall j, u_{j}=u_{j}^{\prime}$ and $\forall j, a_{j} \geq b_{j}$, any induced permutation of the elements satisfies the condition $\forall j, a_{\sigma(j)}^{\prime} \geq_{u} b_{\sigma(j)}^{\prime}$, and $U L O W A_{w}\left(a_{\sigma(1)}^{\prime}, \ldots, a_{\sigma(m)}^{\prime}\right) \geq U L O W A_{w}\left(b_{\sigma(1)}^{\prime}, \ldots, b_{\sigma(m)}^{\prime}\right)$ Then $\operatorname{IULOWA}_{w}(A) \geq \operatorname{IULOWA_{w}}(B)$.

\section{Property 2: The IULOWA operator is commutative} IULOWA $w_{w}\left(\left\langle u_{1}, a_{1}\right\rangle, \ldots .,\left\langle u_{m}, a_{m}\right\rangle\right)=I U L O W A_{w}\left(\left\langle u_{1}^{\prime}, a_{1}^{\prime}\right\rangle, \ldots,\left\langle u_{m}^{\prime}, a_{m}^{\prime}\right\rangle\right)$ where $\left(\left\langle u_{1}^{\prime}, a_{1}^{\prime}\right\rangle, \ldots,\left\langle u_{m}^{\prime}, a_{m}^{\prime}\right\rangle\right)$ is any permutation of the elements in $\left(\left\langle u_{1}, a_{1}\right\rangle, \ldots,\left\langle u_{m}, a_{m}\right\rangle\right)$.

Proof: The IULOWA operator reorders the arguments according to order-inducing variables. Thus, if $A=\left(\left\langle u_{1}, a_{1}\right\rangle, \ldots,\left\langle u_{m}, a_{m}\right\rangle\right)$ is any permutation of $A^{\prime}=\left(\left\langle u_{1}^{\prime}, a_{1}^{\prime}\right\rangle, \ldots,\left\langle u_{m}^{\prime}, a_{m}^{\prime}\right\rangle\right)$, the order induced for $A$ and $A$ ' will be the same.
Therefore, IULOWA $(A)=I U L O W A_{w}\left(A^{\prime}\right)$.

Property 3: The IULOWA operator is idempotent in the sense that IULOWA $\left(\left\langle u_{1}, a_{1}\right\rangle, \ldots .,\left\langle u_{m}, a_{m}\right\rangle\right)=a$, if $\forall j, a_{j}=a$.

Proof: The proof does not depend on the inducing variable, which will take the same value for all the arguments. Then, following the definition of the ULOWA operator, we have that the final step consists in ULOWA $A_{w}\left(a_{m-1}, a_{m}\right)=s_{k}$ where

$$
k=\underset{i \leq p \leq j}{\arg \max }\left\{\operatorname{Sim}\left(s_{p},\left(x_{k}, x_{k}, x_{k}, x_{k}\right)\right)\right\} .
$$

In this case, $p=i=j$ and $s_{k}=s_{i}=a$.

Recursively, we have that $\operatorname{ULOWA}_{w}\left(a_{1}, \ldots ., a_{m}\right)=a$.

Property 4: The IULOWA operator is bounded. That is, for any weighting vector $W$ :

$$
\begin{aligned}
& \min \left(a_{1}, \ldots ., a_{m}\right) \leq \operatorname{IULOWA}\left(\left\langle u_{1}, a_{1}\right\rangle, \ldots .,\left\langle u_{m}, a_{m}\right\rangle\right) \leq \\
& \leq \max \left(a_{1}, \ldots ., a_{m}\right)
\end{aligned}
$$

Proof: Being $s_{j}, s_{i} \in S(j \geq i)$, we have defined the convex combination of these two terms as $C^{2}\left\{w_{i}, b_{i}, i=1,2\right\}=w_{1} \otimes s_{j} \oplus\left(1-w_{1}\right) \otimes s_{i}=s_{k}$. According to Eq. 2, we have that $k=\arg \max _{i \leq p \leq j}\left\{\operatorname{Sim}\left(s_{p}, \delta\right)\right\}$. That is, the resulting label from the combination of two labels $C^{2}\left(s_{i}, s_{j}\right)=s_{k}$ with $i \leq k \leq j$. This means that we cannot obtain a result out of the limits given by the labels that are aggregated at each step.

\section{Order Inducing Variables}

In this section we analyse the distinctive feature of the IULOWA operator against the ULOWA operator, which is the order inducing variable that is used in the reordering process of the linguistic labels. With this type of operator, we are able to deal with complex reordering processes in the analysis of problems in which the highest numerical or linguistic value in $S$ is not the optimal one for the decision maker.

For example, let us consider a linguistic variable describing the temperature. If we are dealing with temperatures of the human body, we assume that the optimal term is Adequate. Thus, if the temperature is higher, the results are not good and it becomes worse if it increases. On the other side, if the temperature is lower, the results are also negative and if it continues decreasing, even worse. So we can use the following order inducing variables: ( $\langle 2$, Very High $\rangle,\langle 5$, High $\rangle,\langle 10$, Medium $\rangle,\langle 4$, Low $\rangle,\langle 1$, Very Low $\rangle)$.

However, when considering the temperature that we want to decide the destination of a trip, we may be interested in a different ordering of the temperatures. For example, a person that prefers to visit places with cold weather would define $(\langle 2$, Very High $\rangle,\langle 4$, High $\rangle,\langle 10$, Medium $\rangle,\langle 8$, Low $\rangle,\langle 6$, Very Low $\rangle)$, whereas for a person that it is not so averse to warm weather, we could 
assume that the ordering is: $(\langle 4$, Very High $\rangle,\langle 8$, High $\rangle$, $\langle 10$, Medium $\rangle,\langle 6$, Low $\rangle,\langle 2$, Very Low $\rangle)$.

Note that, in this example, we associate each linguistic label with an order inducing value. However, in the usual IOWA, the order inducing variables are associated with the considered arguments, such as an order induced by the importance of each argument or its consistency [7]. When using linguistic variables, our suggestion seems to be very natural because the linguistic values have a semantic interpretation for the decision maker.

Moreover, this model regarding the use of order inducing variables with linguistic labels can be extended when using numerical values. In this case, the idea is the same but now we have numbers. That is, situations when, for example, in a numerical scale from 1 to 10 we get that the optimal ranking is not in descending or ascending order. Instead, we have for example: $7>6>$ $8>5>9>4>10>3>2>1$. Note that here ">" means "preferred to". Obviously, in this case we cannot use the OWA aggregation and we need to use the IOWA aggregation.

In addition to the possibility that the decision maker defines his personal policy for inducing the ordering, a further interesting issue arises when dealing with unbalanced sets of terms. As it has been said in the introduction, unbalanced terms permit to define linguistic variables with different granularity and distribution of terms on the positive and on the negative terms. This gives the user a vocabulary with different types of uncertainty on the evaluations. For example, in Figure 1, label $L$ has a larger support that label $V H$ (i.e. the set of values of $\mathrm{x}$ with $\mu_{s_{i}}(x) \geq 0$ is larger).

Consequently, we have labels with different degrees of uncertainty. This uncertainty should be taken into account during the aggregation process, as each label is providing a different amount of information about the evaluated alternative.

In fact, if we consider that both triangular and trapezoidal fuzzy sets can be associated to the labels (as in Figure 1), then, the uncertainty of labels is not only related to their support intervals in the reference domain but also on their kernel (i.e. the set of points of value 1).

Having into account these different features of the definition of the linguistic variable, we propose to use some measure of uncertainty for the linguistic labels as the order inducing criterion of the aggregation. So, the arguments will be ordered by decreasing uncertainty. In this way, the contribution of precise labels is prioritized while the effect of uncertain labels is reduced.

In the literature [16, 20-22], two types of uncertainty are recognized: (1) specificity, related to the measurement of imprecision, which is based on the cardinality of the set, and (2) fuzziness, or entropy, which measures the vagueness of the set as a result of having imprecise boundaries.

Definition 2. Measure of Specificity [22]:

Let $X$ be a set and let $[0,1]^{X}$ be the class of fuzzy sets on $X$. A measure of specificity is a function $S p$ : $[0,1]^{X} \rightarrow[0,1]$ such that:

1. $\operatorname{Sp}(\varnothing)=0$
2. $\operatorname{Sp}(\mu)=1$ if and only if $\mu$ is a singleton

3. If $\mu$ and $\eta$ are normal fuzzy sets in $X$ and $\mu \subset \eta$, then $\operatorname{Sp}(\mu) \geq S p(\eta)$.

In [20], the following specificity measure, for a fuzzy set $A$ defined on $X$, is defined as a generalization of other previous formulations:

$$
\operatorname{Sp}(A)=T\left(\alpha_{\text {sup }}, N\left(\int_{0}^{\alpha_{\text {sup }}} M\left(A_{\alpha}\right) d \alpha\right)\right)
$$

being $T$ a T-norm, $N$ a negation operator and $M$ a fuzzy measure.

In Eq. 7 a special case of Eq. 6 is given, by considering the T-norm $\min$, the standard negation $N=1-x$ and the Lebesgue-Stieltjes fuzzy measure $M([a, b])=b-a$. Taking a normalized fuzzy set (with $\alpha_{\text {sup }}=1$ ), and with these parameters, the specificity can be calculated as:

$$
\operatorname{Sp}(A)=1-\frac{\text { area under } A}{b-a}
$$

Definition 3. Measure of Fuzziness [23]:

Let $X$ be a set and let $[0,1]^{X}$ be the class of fuzzy sets on $X$. A measure of fuzziness is a function $\mathrm{Fz}$ : $[0,1]^{X} \rightarrow[0,1]$ such that:

1. $\quad F_{z}(A)=0$ iff $A$ is a crisp set

2. $F z(A)=1$ iff $\forall x \in X, A(x)=1 / 2$

3. $F z(A) \leq F z(B)$ if $A$ is less fuzzy than $B$, i.e. $A(x) \leq B(x) \leq 1 / 2$ or $A(x) \geq B(x) \geq 1 / 2$ for every $x \in X$.

The most common way to calculate the fuzziness is in terms of the lack of distinction between the fuzzy set $A$ and its complement $A^{C}$. A general definition of this type of fuzziness measures is based on an aggregation operator $h$ and a distance function $d$, as:

$$
F z(A)=h_{x \in A}\left(d\left(A(x), A^{C}(x)\right)\right)
$$

For the case of continuous domains, and taking the standard negation operation and the Hamming distance, Eq. 8 corresponds to:

$$
F z(A)=1-\frac{1}{b-a} \int_{a}^{b}|2 \cdot A(x)-1|
$$

Specificity and fuzziness refer to two different characteristics of fuzzy sets. Specificity (or its counterpart non-specificity [24]) measures the degree of truth of the sentence "containing just one element". Fuzziness is measuring the difference from a crisp set. For decision making purposes, it seems desirable to have labels that correspond to single elements, rather than to large sets of values, which may difficult the selection of the appropriate alternative. For this reason, we propose to use a measure of specificity as order inducing variable in 
the aggregation of linguistic terms that are qualifying a set of alternatives in a decision making process.

In case of having ties between different terms with the same specificity, a second ordering criterion may be the fuzziness associated to the set. An increasing ordering of fuzziness will be considered, as we prefer the terms with less uncertainty. If this second criterion also leads to some ties, a decreasing ordering on the preference scale associated to the terms can be considered (decreasing order of the linguistic labels in $S$ ). In Fig. 2, we show two fuzzy sets with the same specificity $(\operatorname{Sp}(A)=\operatorname{Sp}(B)=0.9)$ according to Eq. 7 but different fuzziness (Eq. 9) $(F z(A)=0.1$ and $F z(B)=0.05)$. In this example, the set $A$ is more fuzzy than $B$, so $B$ is preferred.

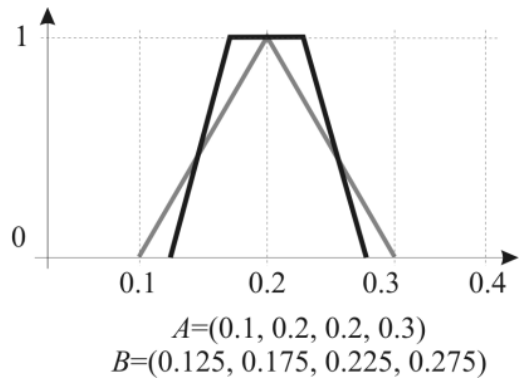

Fig. 2: Two fuzzy sets with the same specificity and different fuzziness

According to this procedure, the IULOWA operator applied to a set or arguments $\left\{a_{1}, \ldots, a_{m}\right\}$ we calculate the induced aggregation according to the uncertainty of those $m$ terms using IULOWA (5) where $B$ is the induced ordering vector, such that $B=\left(b_{1}, b_{2}, \ldots, b_{m}\right)$ satisfies these conditions:

- $\quad \forall k 1 \leq k<m \quad S p\left(b_{k}\right) \geq S p\left(b_{k+1}\right)$

- $\forall k \quad 1 \leq k<m$ if $\operatorname{Sp}\left(b_{k}\right)=\operatorname{Sp}\left(b_{k+1}\right)$ then

$$
F z\left(b_{k}\right) \leq F z\left(b_{k+1}\right)
$$

- $\forall k \quad 1 \leq k<m$ if $\operatorname{Sp}\left(b_{k}\right)=\operatorname{Sp}\left(b_{k+1}\right)$ and

$$
F z\left(b_{k}\right)=F z\left(b_{k+1}\right) \text { then } b_{k}>b_{k+1} \text { according to the }
$$

linguistic scale $S$

Notice that if the set of terms corresponds to crisp numbers, IULOWA is reduced to the OWA operator.

OWA weights $w_{i}$ are used to define different conjunction/disjunction aggregation models [19]. As proposed by $[3,6]$, the procedure of inclusion of an additional variable into the OWA may involve also the transformation of the set of weights.

We also propose to modify the set of weights associated to the arguments by taking into consideration the specificity of the values that are aggregated. In this way, the more specific values should have a higher weight, whereas the less specific terms (less reliable) should have a lower weight.
Considering the family of fuzzy quantifiers proposed by Yager [4], the set of weights is obtained by the expression:

$$
w_{k}=Q\left(\frac{S(k)}{S(n)}\right)-Q\left(\frac{S(k-1)}{S(n)}\right) \text {, }
$$

where $S(k)=\sum_{i=1}^{k} u_{\sigma(i)}$ and $\sigma$ is the permutation according to the order inducing procedure established before (Eq. 10).

The properties of the quantifier function must be taken into account in order to generate a coherent set of weights for the OWA operator. Considering the usual quantifier $Q(r)=r^{a}$ [4], when $a \in[0,1]$ then the weighting function is concave, satisfying that the largest the specificity, the higher the weight $w_{k}$ of the corresponding argument [6]. It is worth to note that with $a \in[0,1]$ the aggregation policy is disjunctive, facilitating the replaceability of uncertain evaluations by the most specific (and less fuzzy) available values.

\section{Examples}

In this section three examples are analysed. The first is an example to show how the sorting of terms is performed according to the measures of specificity and fuzziness. The second example studies how the specificity can influence the generation of the aggregation weights. Finally, the third example is devoted to use the IULOWA operator in a decision problem in which research papers have to be evaluated.

\subsection{Order induction example}

As previously stated, the order of aggregation of the linguistic fuzzy terms is found by sorting the labels we want to aggregate according to their specificity. Ties between terms with the same measure of specificity are solved by taking into account the fuzziness of each fuzzy set. If that measure still has any ties, terms will finally be ordered according to the index (i.e. position) of the terms in the linguistic scale $S$, (assuming that the highest positions are the best).

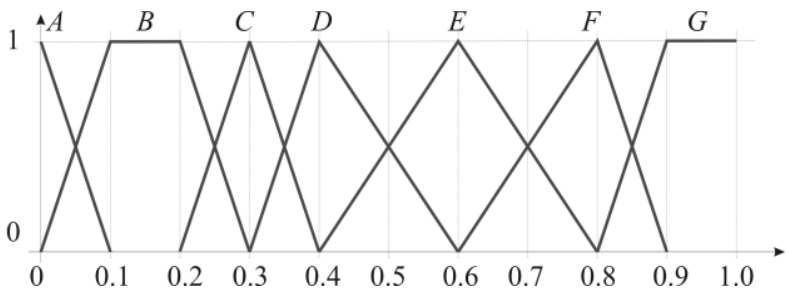

Fig. 3: Linguistic variable with 7 terms (test 1)

In this example we will show how would be sorted the terms depicted in Fig. 3. The information regarding each of the terms necessary to conduct that sorting procedure is indicated in Table 1. Specificity is calculated following Eq. 7, whereas fuzziness is obtained using Eq. 9. 


\begin{tabular}{|c|c|c|c|c|}
\hline Term & Definition & Index & Specificity & Fuzziness \\
\hline$A$ & $(0.0,0.0,0.0,0.1)$ & 0 & 0.95 & 0.05 \\
$B$ & $(0.0,0.1,0.2,0.3)$ & 1 & 0.8 & 0.10 \\
$C$ & $(0.2,0.3,0.3,0.4)$ & 2 & 0.9 & 0.10 \\
$D$ & $(0.3,0.4,0.4,0.6)$ & 3 & 0.85 & 0.15 \\
$E$ & $(0.4,0.6,0.6,0.8)$ & 4 & 0.8 & 0.20 \\
$F$ & $(0.6,0.8,0.8,0.9)$ & 5 & 0.85 & 0.15 \\
$G$ & $(0.8,0.9,1.0,1.0)$ & 6 & 0.85 & 0.05 \\
\hline
\end{tabular}

Table 1: Uncertainty measures for the terms in test 1

Taking into account the specificity, the labels are ordered as $A>C>(D, F, G)>(B, E)$. Note that there are two ties: the first one between $D, F$ and $G$ (with $S p=0.85$ ), and the second one between $B$ and $E(S p=0.8)$. Using the fuzziness measure to solve the ties, we put $G(\mathrm{Fz}=0.05)$ before $D$ and $F(F z=0.15)$ in the first tie, while $B\left(F_{z}=0.10\right)$ goes before $E\left(F_{z}=0.20\right)$ in the second tie. As we can see, the measure of fuzziness is still unable to decide the order between $D$ and $F$, so we use the index of the terms to decide the position, putting $F$ (index $=5$ ) before $D$ (index $=3$ ). Thus, the induced order according to the procedure proposed in this paper (Eq. 10) is: $A>C>G>F>D>B>E$.

\subsection{Weight generation example}

Weight generation is conducted by using fuzzy quantifiers and taking into account the measure of specificity of each term we want to aggregate. We have tested how the specificity of the terms modifies the weights that are finally associated to each argument.

We will use the fuzzy quantifier $Q(r)=r^{a}$ to generate the weighting policy for the OWA operator. Table 2 shows the weights obtained without taking into account the specificities. Tests have been done considering several values of the parameter $a$, ranging from 0.1 (where we mostly base the result on the first argument) to 1 (which corresponds to an arithmetic average of the arguments, as the weights are equal for all the values).

\begin{tabular}{|c|c|}
\hline$a^{*}$ & Weights \\
\hline 0.1 & $(0.851,0.061,0.038,0.028,0.022)$ \\
0.25 & $(0.668,0.127,0.085,0.066,0.054)$ \\
0.5 & $(0.447,0.185,0.142,0.120,0.106)$ \\
0.75 & $(0.299,0.204,0.179,0.164,0.154)$ \\
1 & $(0.200,0.200,0.200,0.200,0.200)$ \\
\hline \\
Talues in the quantifier function \\
Table 2: Weights obtained without specificity
\end{tabular}

Two tests have been done. The first one is based on the linguistic variable with 7 terms represented in Fig. 3. We consider the generation of weights for the values $(A, C, F, B, B)$ with specificities $(0.95,0.9,0.85,0.8,0.8)$ respectively. The results are shown in Table 3.

\begin{tabular}{|c|c|}
\hline$a$ & Weights \\
\hline 0.1 & $(0.860,0.059,0.036,0.025,0.020)$ \\
0.25 & $(0.686,0.124,0.080,0.060,0.050)$ \\
0.5 & $(0.470,0.186,0.136,0.110,0.098)$ \\
0.75 & $(0.322,0.209,0.174,0.152,0.143)$ \\
1 & $(0.221,0.209,0.198,0.186,0.186)$ \\
\hline \multicolumn{2}{|c}{ Table 3: Weights obtained in test 1 }
\end{tabular}

In this test, the specificities of the terms that are aggregated are very similar among them. For this reason, the weights in Table 3 are quite similar to the ones obtained in Table 2 when specificity was not considered. This illustrates that when the specificity (i.e. confidence) on the terms is similar, the weights are almost not modified.

For the second test we have used another set of terms with different degrees of specificity, as shown in Fig. 4. In this case, we aggregate the values $(E, B, B, C, C)$ with specificities $(0.95,0.8,0.8,0.5,0.5)$ respectively.

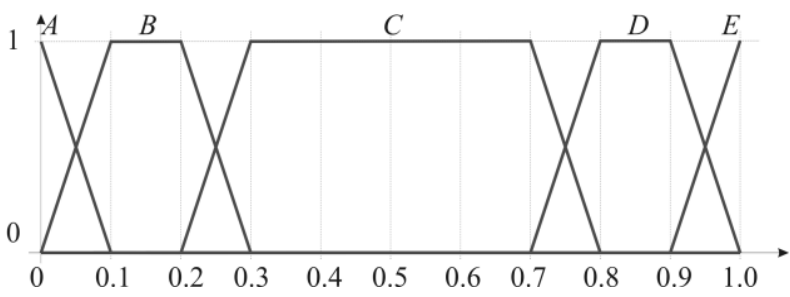

Fig. 4: Linguistic variable with 5 terms (test 2)

\begin{tabular}{|c|c|}
\hline$a$ & Weights \\
\hline 0.1 & $(0.876,0.056,0.036,0.017,0.015)$ \\
0.25 & $(0.719,0.119,0.083,0.042,0.037)$ \\
0.5 & $(0.517,0.187,0.145,0.079,0.072)$ \\
0.75 & $(0.372,0.216,0.192,0.112,0.108)$ \\
1 & $(0.268,0.225,0.225,0.141,0.141)$ \\
\hline \multicolumn{2}{|c|}{ Table 4: Weights obtained in test 2 }
\end{tabular}

In this second test the two last terms have a low specificity $(0.5)$ considering the rest of values for the first three terms $(0.95$ to 0.8$)$. The results given in Table 4 show that this difference affects the weights as expected, giving more weight to the less uncertain terms. We can see a notable increase of the global weight of the first three terms and a decrease of the weight of the last two terms.

\subsection{A complete example of IULOWA}

Let us consider that the terms in Fig. 4 represent the evaluations the reviewers can give when reviewing a research paper, being $\mathrm{A}=$ "Disaster", $\mathrm{B}=$ " $\mathrm{Bad}$ ", $\mathrm{C}=$ "Normal", $\mathrm{D}=$ "Good", $\mathrm{E}=$ "Excellent". We consider that the reviewers who give the most precise evaluations (such as "Excellent" or "Disaster") are the ones with greatest knowledge in the paper's content or, at least, are more convinced to decide that extreme score. This is why we want to increase the weight we give to those specific evaluations rather than the ones that just give a "Normal" rating, which is quite unspecific. Thus, we are using an order inducing process because the information is reordered according to an additional criterion different from the values of the arguments.

In this example, we have five evaluations to aggregate in order to decide a global score for a paper, using the IULOWA operator. Those evaluations are $(\mathrm{C}, \mathrm{C}, \mathrm{D}$, $\mathrm{A}, \mathrm{C})$.

The first step is to calculate the order in which the evaluations will be aggregated according to the measures of uncertainty given in this paper (Eq. 7 and 
Eq. 9). Those measures are given in Table 5. The order of the arguments is, then, (A, D, C, C, C).

\begin{tabular}{|c|c|c|c|c|}
\hline Term & Definition & Index & Specificity & Fuzziness \\
\hline$A$ & $(0.0,0.0,0.0,0.1)$ & 0 & 0.95 & 0.05 \\
$B$ & $(0.0,0.1,0.2,0.3)$ & 1 & 0.8 & 0.10 \\
$C$ & $(0.2,0.3,0.7,0.8)$ & 2 & 0.5 & 0.10 \\
$D$ & $(0.7,0.8,0.9,1.0)$ & 3 & 0.8 & 0.10 \\
$E$ & $(0.9,1.0,1.0,1.0)$ & 4 & 0.95 & 0.05 \\
\hline
\end{tabular}

Table 5: Term set uncertainty measures

After sorting the arguments, we have used the $S p$ measure (Eq. 7) to generate the weighting vector as done in the previous example (Eq. 11), using $Q(r)=r^{a}$ with $a=0.75$. The resulting weighting vector is $\mathrm{W}=(0.398$, $0.231,0.130,0.123,0.118$ ).

The IULOWA aggregation begins following (Eq. 5):

$$
\begin{aligned}
& \text { IULOWA }(A, D, C, C, C)=W \cdot B^{T}= \\
& =C^{5}\left\{w_{k}, b_{k}, k=1, . ., 5\right\}= \\
& =0.398 \otimes A \oplus(1-0.398) \otimes C^{4}\left\{\beta_{h}, b_{h}, h=2, . ., 5\right\}
\end{aligned}
$$

Now, we have to evaluate $C^{4}$ as:

$$
\begin{aligned}
& \quad C^{4}\left\{\beta_{h}, b_{h}, h=2, . .5\right\}= \\
& \quad\left(\left(\frac{0.231}{0.602}\right) \otimes D \oplus\left(1-\frac{0.231}{0.602}\right) \otimes C^{3}\left\{\beta_{h}, b_{h}, h=3, . ., 5\right\}\right) \\
& \text { where } \\
& C^{3}\left\{\beta_{h}, b_{h}, h=3, . ., 5\right\}= \\
& \left(\left(\frac{0.130}{0.371}\right) \otimes C \oplus\left(1-\frac{0.130}{0.371}\right) \otimes C^{2}\left\{\beta_{h}, b_{h}, h=4, . .5\right\}\right)
\end{aligned}
$$$$
\text { , where }
$$

In the last step, $C^{2}$ is calculated as follows:

$$
\begin{aligned}
& C^{2}\left\{\beta_{h}, b_{h}, h=4, . ., 5\right\}= \\
& \left(\left(\frac{0.123}{0.241}\right) \otimes C \oplus\left(1-\frac{0.123}{0.241}\right) \otimes C\right)=s_{k}
\end{aligned}
$$

Now, the two terms to aggregate are $s_{j}=C$ and $s_{i}=C$. In this case it is trivial to find the aggregation result: $C$. Then, we continue with the same approach. The two terms to aggregate to obtain $C^{3}$ are the same: $s_{j}=C$ and $s_{i}=C$, obtaining again a $C$ score.

The next terms to aggregate, to calculate $C^{A}$, are $s_{j}=D$ and $s_{i}=C$. Following the ULOWA convex combination (Eq. 3), we find an intermediate point between these two terms, which is calculated taking into account the centers of gravity of the corresponding fuzzy sets as well as their weights. This point is $\delta=(0.634,0.634,0.634,0.634)$. It is necessary then to calculate the similarity of that intermediate point with all the terms comprised between $C$ and $D$. The similarities of $C$ and $D$ with $\delta$ are 0.78 and 0.744 respectively, so the aggregation result of $C$ and $D$ is $C$.

The final step is to calculate $C^{5}$ by aggregating $A$ and $C$. The intermediate point between $A$ and $C$ is in this case $\delta=(0.398,0.398,0.398,0.398)$. The similarities of $A, B$ (which is between $A$ and $C$ ) and $C$ with $\delta$ are $0.626,0.748$ and 0.736 respectively, so the aggregation result of $A$ and $C$, and the final aggregation result, is $B$.
Note that if the same process is done without taking into account the specificities when sorting the labels and calculating the weights (as in ULOWA), the aggregation order would have been $(\mathrm{D}, \mathrm{C}, \mathrm{C}, \mathrm{C}, \mathrm{A})$ and the weighting vector $(0.299,0.204,0.179,0.164,0.154)$. In this case we obtain that the final aggregation result is $C$. This is not adequate if we want to increase the importance of the most precise evaluations, $A$ in this case.

\section{Conclusions and future work}

The use of the IULOWA operator permits to deal with complex reordering processes by using order inducing variables. Thus, we are able to deal with problems where the highest results may not be the optimal ones, because there are other criteria that influence the ordering of the arguments. In particular, the IOWA operator defined in [5] has been extended to the case of dealing with unbalanced linguistic variables that use unbalanced fuzzy sets. Unbalanced sets of terms introduce the property of dealing with different degrees of uncertainty in the values that are aggregated.

The paper proposed a procedure to use the measurement of uncertainty as order inducing criterion in IULOWA. With this approach, the decision is based on the less uncertain values, which have more confidence from the user point of view. The concept of minimum uncertainty is interpreted as having the maximum specificity and the minimum fuzziness, two well-known measures in fuzzy theory. Ties are solved by taking as preference degree the scale of evaluation.

It can be easily seen that we have defined a more general framework that includes the ULOWA operator when all the terms have the same specificity and fuzziness. It is also reduced to the LOWA operator if the terms are balanced. In fact, the IULOWA operator provides a wide range of families of unbalanced linguistic aggregation operators following the methodology used in the OWA literature $[1,16,18]$. For example, we have the unbalanced linguistic minimum, the unbalanced linguistic maximum, the unbalanced linguistic average, the unbalanced linguistic weighted average or the unbalanced linguistic median.

The paper also shows that it is interesting to modify the weighting policy according to the level of uncertainty to make a coherent aggregation of the values.

In future research, we expect to develop further aggregation operators for unbalanced linguistic variables, for example, including the possibility of introducing the importance of the variables. We will also study the applicability of this approach in several areas such as decision making theory or online recommender systems.

\section{Acknowledgements}

This work has been supported by the Universitat Rovira i Virgili (a pre-doctoral grant of L. Marin) and the Spanish Ministry of Science and Innovation (DAMASK project, Data mining algorithms with semantic knowledge, TIN2009-11005) and the Spanish Government (Plan E, Spanish Economy and Employment Stimulation Plan). 


\section{References}

[1] M. Pazzani and D. Billsus, Learning and revising user profiles: The Identification of Interesting Web Sites, Mach. Learn., 27:313-331, 1997.

[2] J.M. Merigó and M. Casanovas, Induced aggregation operators in decision making with the Dempster-Shafer belief structure, International Journal of Intelligent Systems, 24:934-954, 2009.

[3] V. Torra and Y. Narukawa, Modeling Decisions: Information Fusion and Aggregation Operators, Springer Berlin Heidelberg, 2007.

[4] R.R. Yager, On ordered weighted averaging aggregation. Operators in multicriteria decisionmaking, IEEE Transactions on Systems, Man and Cybernetics, 18:183-190, 1988.

[5] R.R. Yager and D.P. Filev, Induced Ordered Weighted Averaging operators, IEEE Transactions on Systems, Man, and Cybernetics, Part B: Cybernetics, 29:141-150, 1999.

[6] F.J. Cabrerizo, I.J. Pérez and E. Herrera-Viedma, Managing the consensus in group decision making in an unbalanced fuzzy linguistic context with incomplete information, Knowledge-Based Systems, 23:169-181, 2010.

[7] J.M. Merigó and M. Casanovas, Decision making with distance measures and linguistic aggregation 0operators, International Journal of Fuzzy Systems, 12:190-198, 2010.

[8] J.M. Merigó and A.M. Gil-Lafuente, The induced generalized OWA operator, Information Sciences, 179:729-741, 2009.

[9] J.M. Merigó, M. Casanovas and L. MartÍnez, Linguistic aggregation operators for linguistic decision making based on the dempster-shafer theory of evidence, International Journal of Uncertainty, Fuzziness and Knowlege-Based Systems, 18:287-304, 2010.

[10] F. Herrera and L. Martínez, A 2-tuple fuzzy linguistic representation model for computing withwords, IEEE Transactions on Fuzzy Systems, 8:746 - 752, 2000.

[11] F. Herrera, E. Herrera-Viedma and L. Martínez, A fuzzy linguistic methodology to deal with unbalanced linguistic term sets, IEEE Transactions on Fuzzy Systems, 16:354-370, 2008.

[12] L.A. Zadeh, The concept of a linguistic variable and its application to approximate reasoning-I, Information Sciences, 8:199-249, 1975.

[13] G. Beliakov, A. Pradera and T. Calvo, Aggregation Functions: A Guide for Practitioners, Springer-Verlag, Berlin, 2007.

[14] Z. Xu, An interactive approach to multiple attribute group decision making with multigranular uncertain linguistic information, Group Decision and Negotiation, 18:119-145, 2009.

[15] D. Isern, L. Marin, A. Valls and A. Moreno, The unbalanced linguistic ordered weighted averaging operator, in: Proc. of. IEEE International Conference on Fuzzy Systems, FUZZ-IEEE 2010, Barcelona, Catalonia, pages 3063-3070, IEEE Computer Society, 2010 .
[16] P. Bonissone and K. Decker, Selecting uncertainty calculi and granularity: an experiment in trading-off precision and complexity, in: L.N. Kanal, J.F. Lemmer (eds.), Proc. of. First Annual Conference on Uncertainty in Artificial Intelligence, UAI-85, New York, NY, pages 217-248, Elsevier Science, 1985.

[17] F. Herrera, E. Herrera-Viedma and J.L. Verdegay, Direct approach processes in group decision making using linguistic OWA operators Fuzzy Sets and Systems, 79:175-190 1996.

[18] J.M. Merigó and M. Casanovas, The uncertain induced quasi-arithmetic OWA operator, International Journal of Intelligent Systems, 26:1-24, 2011.

[19] R.R. Yager, Including importances in OWA aggregations using fuzzy systems modeling, IEEE Transactions on Fuzzy Systems, 6:286-294, 1988.

[20] L. Garmendia, R.R. Yager, E. Trillas and A. Salvador, A t-norm based specificity for fuzzy sets on compact domains, International Journal of General Systems, 35:687-698, 2006.

[21] G.J. Klir, Developments in uncertainty-based information, in: C.Y. Marshall (Ed.) Advances in Computers, Elsevier, 1993, pp. 255-332.

[22] R.R. Yager, Ordinal measures of specificity, International Journal of General Systems, 17:57-72, 1990.

[23] A. De Luca and S. Termini, A definition of a nonprobabilistic entropy in the setting of fuzzy sets theory, Information and Control, 20:301-312, 1972.

[24] G.J. Klir and B. Yuan, On nonspecificity of fuzzy sets with continuous membership functions, in: Proc. of. 15th International Conf. on Systems, Man, and Cybernetics, Vancouver, Canada, pages 25-29, IEEE Computer Society, 1995. 